\title{
Brugada Pattern Type 2 Diagnosis Unmasked by Aspiration Pneumonia
}

\author{
Yasar Sattar $^{1}$, Waqas Ullah ${ }^{2}$, Syeda Ramsha Zaidi ${ }^{3}$, Talal Almas ${ }^{4}$, M. Chadi Alraies ${ }^{5}$ \\ 1. Internal Medicine, Icahn School of Medicine at Mount Sinai, New York City, USA 2. Internal Medicine, Abington \\ Hospital-Jefferson Health, Abington, USA 3. Internal Medicine, St. Mary Mercy Hospital, Livonia, USA 4. Internal \\ Medicine, Royal College of Surgeons in Ireland, Dublin, IRL 5. Cardiology, Detroit Medical Center, Detroit, USA
}

Corresponding author: Yasar Sattar, sattary@nychhc.org

\begin{abstract}
Brugada syndrome (BrS) is a rare autosomal dominant mutation affecting sodium channels. Electrocardiography can show two Brugada patterns (BrP). Type $1 \mathrm{BrP}$ usually causes sudden cardiac arrest (SCA). Type 2 BrP can appear during circumstances that result in delayed sodium channel opening, such as fever, pneumonia, or use of sodium channel blockers. Patients with type 2 BrP often have underlying type 1 $\mathrm{BrP}$; this can be confirmed by an ajmaline challenge test. We describe the case of a patient who presented with SCA. He later had an interval type $2 \mathrm{BrP}$ secondary to aspiration pneumonia, followed by type $1 \mathrm{BrP}$ pattern confirmed by an ajmaline challenge test. The patient ultimately underwent implantable cardiac defibrillator placement to prevent future SCA.
\end{abstract}

Categories: Cardiology, Emergency Medicine, Internal Medicine

Keywords: brugada syndrome, brugada pattern, aspiration pneumonia

\section{Introduction}

Brugada syndrome (BrS) was initially described by Pedro and Joseph Brugada in 1992 [1]. BrS is an autosomal dominant mutation affecting sodium channels, which can provoke sudden cardiac arrest (SCA) owing to ventricular arrhythmia [1,2]. Electrocardiographic (ECG) characteristics of BrS can follow one of two Brugada patterns (BrP): the type 1 pattern features a coved-type ST segment, while the type 2 BrP features a saddleback ST segment in precordial leads V1-V3 [3]. Patients with BrS can present with a normal initial ECG; however, BrP can be unmasked by specific stressors, such as infection, fever, psychosis, medications including sodium channel blockers, propranolol, tricyclic antidepressants, and illicit drugs such as cocaine [4]. We describe a case of a patient with BrS who presented with SCA, followed by an interval appearance of type $2 \mathrm{BrP}$ in the setting of aspiration pneumonia.

\section{Case Presentation}

Received 05/07/2020

Review began 05/19/2020 Review ended 05/20/2020 Published 05/28/2020

\section{(c) Copyright 2020}

Sattar et al. This is an open access article distributed under the terms of the Creative Commons Attribution License CC-BY 4.0., which permits unrestricted use, distribution, and reproduction in any medium, provided the original author and source are credited.
A 21-year-old man with no significant past medical history was brought to the emergency department (ED) by emergency medical services for SCA with ventricular fibrillation (VF) in the field. He was ultimately shocked four times and given two rounds of epinephrine prior to the return of spontaneous circulation. Upon arrival at the ED, he had regained pulses and was started on minimal vasopressors and low tidal volume minute ventilation (LTVMV). His initial vital signs and breathing status were stable. An initial electrocardiogram (ECG) was performed, which revealed a sinus rhythm (Figure 1).

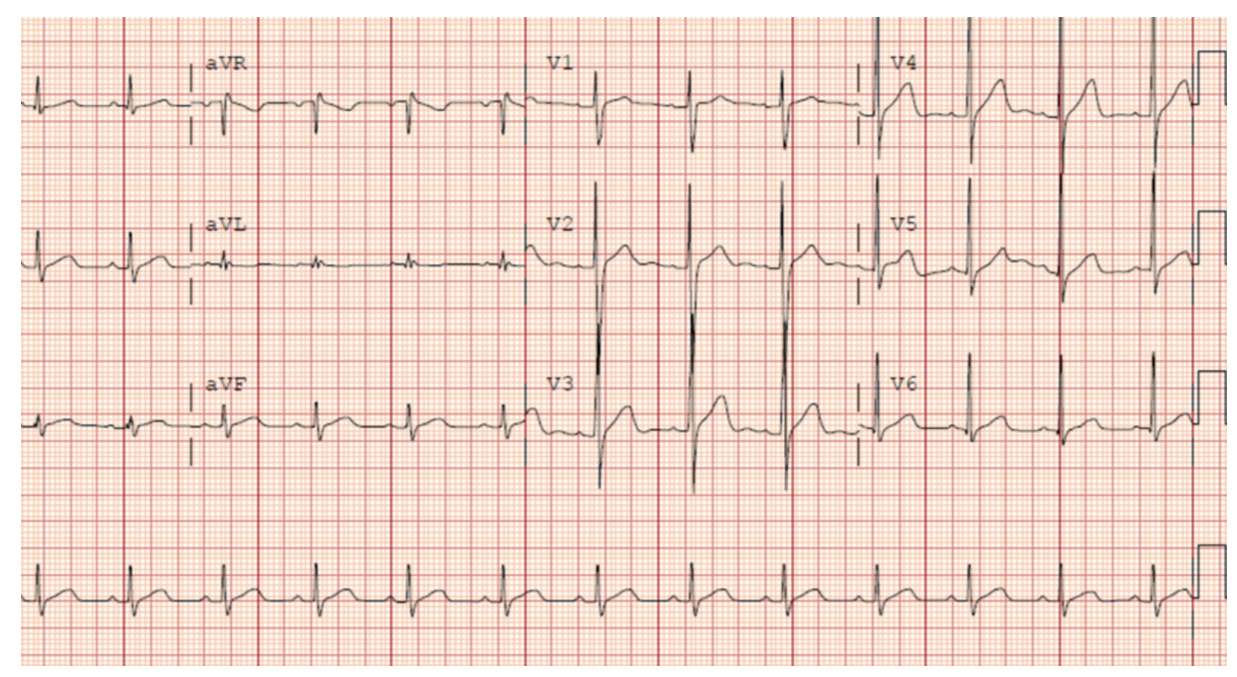




\section{Cureus}

FIGURE 1: Electrocardiogram revealing normal sinus rhythm, no signs of ischemia, and normal intervals.

The findings of his physical examination were non-significant; he had intact brainstem reflexes and normal cardiopulmonary exam on ventilation. After one day in the intensive care unit (ICU), his vital signs were as follows: temperature, $101^{\circ} \mathrm{F}$; blood pressure, $110 / 70 \mathrm{mmHg}$; heart rate, 90 beats/minute; respiratory rate, 22 breaths/minute; and oxygen saturation, 98\% on LTVMV. A repeat ECG during an interval fever episode revealed a saddleback T wave in precordial leads V1-V2 consistent with type $2 \mathrm{BrP}$ (Figure 2).

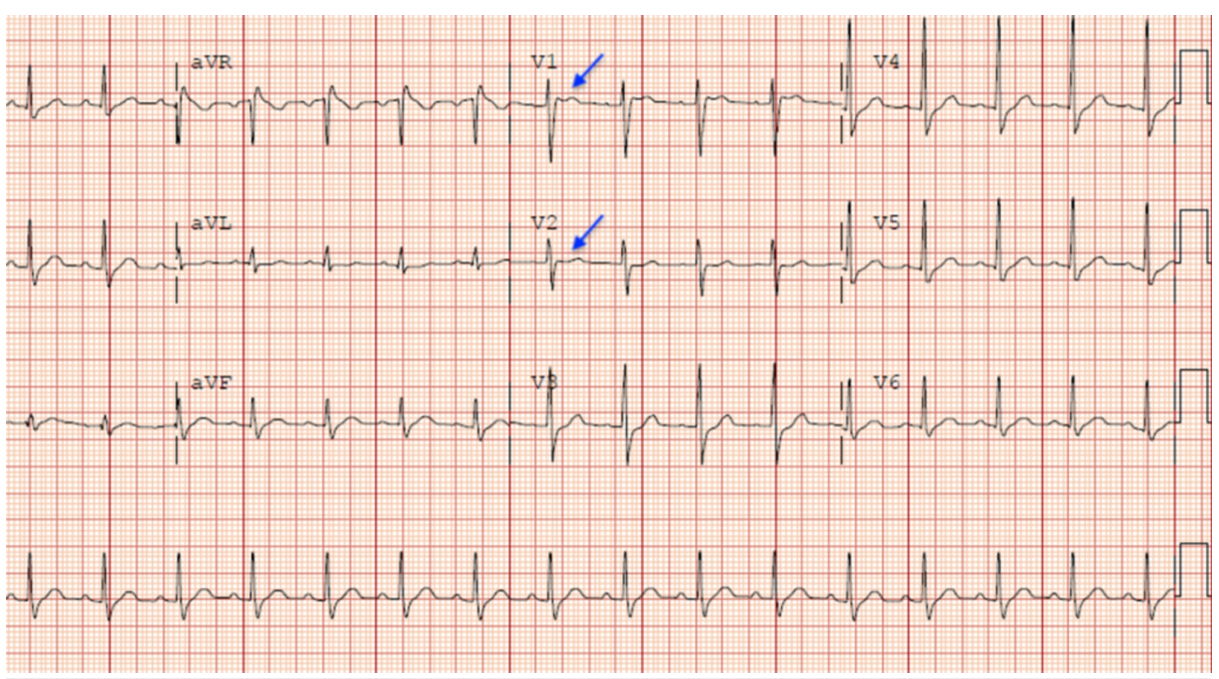

FIGURE 2: Electrocardiogram revealing type 2 Brugada pattern showing saddleback ST pattern in V1-V2 (arrows).

CT of the chest showed patchy bilateral infiltrates consistent with aspiration pneumonia (Figure 3).

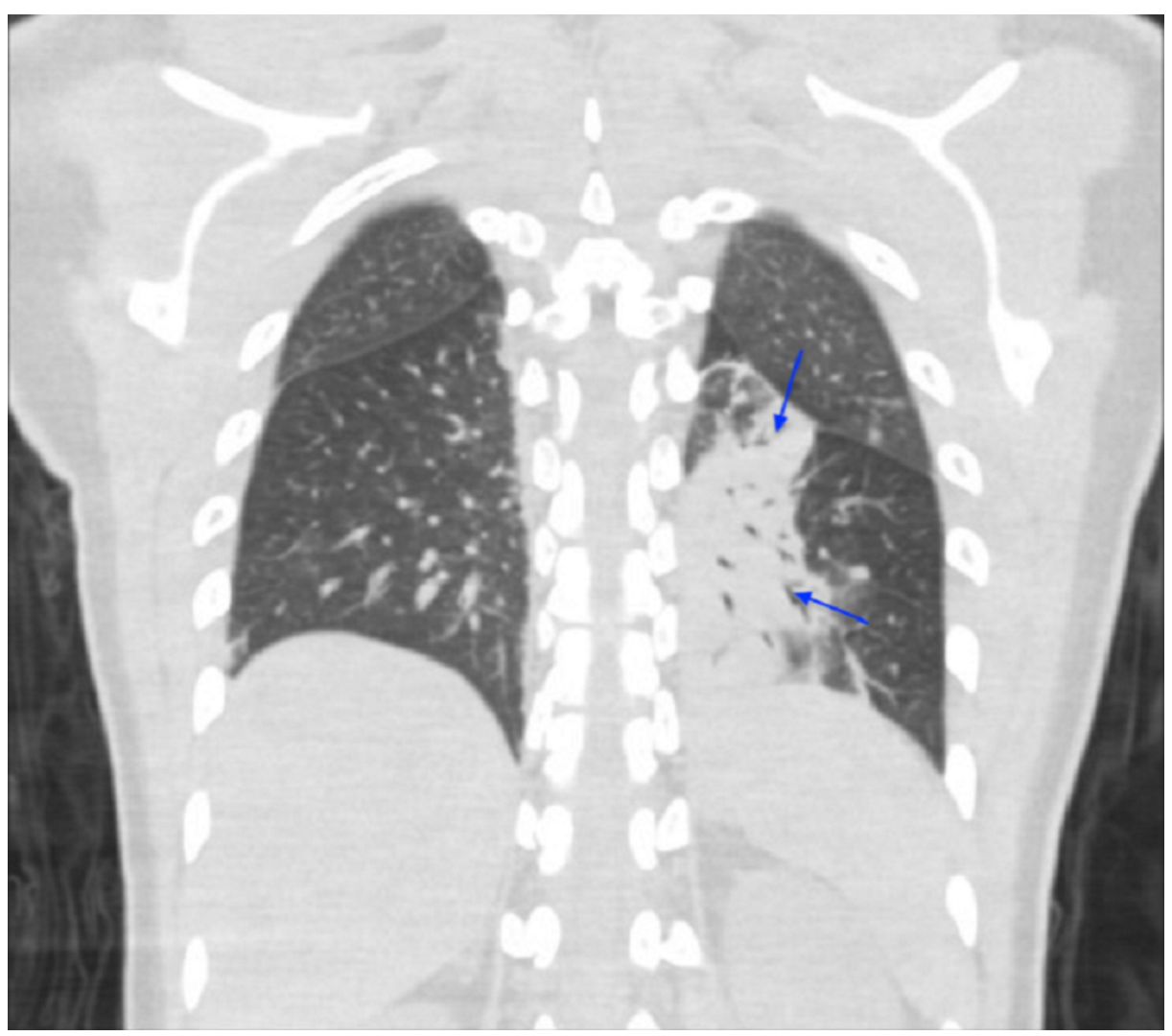



ejection fraction (LVEF) of 20\%; improvement to normal LVEF was noted within 24 hours (Video 1).

\section{VIDEO 1: Normal four-chamber transthoracic echocardiography showing normal chambers and valve motion.}

View video here: https://vimeo.com/419847049

Because of the patient's SCA and ECG findings, he was presumed to have type 2 BrP provoked by fever and pneumonia. The patient was initially started on low-dose norepinephrine, fluids, vancomycin/cefepime, and LTVMV, with successful weaning.

The patient was monitored via follow-up in the cardiology clinic, where he tested positive to an ajmaline challenge test for type $1 \mathrm{BrP}$. The patient underwent implantable cardiac defibrillator (ICD) placement during outpatient follow-up.

\section{Discussion}

\section{Pathophysiology}

$\mathrm{BrS}$ is an autosomal mutation affecting sodium channels. The genetic mutation in BrS commonly affects epicardial and M cells of the heart. Some of the common mutations involve the genes SCN10A and SCN5A [5]. Mutations in sodium channels lead to a decrease in the inward current of sodium, causing shorter action potentials [6]. The shortening of action potentials due to mutations is further enhanced by an increased counter outward current of sodium, as found in the right ventricular outflow tract in some patients. This causes reduced initial depolarization and delayed phase 2 due to reduced co-activation of calcium channels. These voltage changes of sodium channels appear as ST-segment elevation and T-wave inversion seen in the right precordial leads [7]. The effect of mutated sodium channels can be further enhanced by risk factors, such as fever, psychosis, medications including sodium channel blockers, propranolol, tricyclic antidepressants, or illicit drugs like cocaine.

\section{Clinical presentation and diagnostic criteria}

While the clinical manifestations of BrS may vary, BrP is usually asymptomatic. The presence of a type $1 \mathrm{BrP}$ subtype portends to a worse prognosis and predisposes to debilitating clinical outcomes such as SCA. Clinical presentation of BrS can also include atrial tachyarrhythmias and life-threatening ventricular arrhythmias, including VF and ventricular tachycardia (VT). Syncope or SCA can be one of the sequelae of tachyarrhythmias, and establishing a diagnosis of BrS mainly involves clinical symptoms and ECG findings.

In 2005, the Heart Rhythm Society (HRS) and the European Heart Rhythm Association (EHRA) stipulated specific criteria for BrP types 1 and 2 [8]. The HRS and EHRA stated that type $1 \mathrm{BrP}$ includes coved-type STsegment elevations in precordial leads (V1-V2) with or without the use of sodium channel blockers [9]. The criteria for type $2 \mathrm{BrS}$ included a saddleback-type ST-segment elevation in more than one precordial lead (V1-V2) at baseline but conversion to coved type in similar precordial leads upon administration of sodium channel blockers [9]. To meet the requisite criteria, patients must also demonstrate at least one of the following: family history of type $1 \mathrm{BrP} / \mathrm{BrS}$, VF, VT, or nocturnal agonal respiration [9]. Initial ECG findings are often unremarkable in $\mathrm{BrP}$ and $\mathrm{BrS}$, and risk factor such as infections, fever, psychosis, medications (e.g., sodium channel blockers, propranolol, tricyclic antidepressants), and illicit drugs (e.g., cocaine) should prompt more frequent ECG workup for BrS and BrP [3].

BrP can be divided into three subtypes, each varying with pertinence to the typical ECG findings that they elicit. It is imperative to note, however, that findings compatible with BrP type 1 are necessary to yield a diagnosis of BrS, owing primarily to the fact that ECG findings inherent to types 2 and 3 are also commonly found in patients who have no indication of a BrS [10]. Thus, while type 2 and 3 findings can insinuate a diagnosis of BrS, challenge testing is necessary for establishing and confirming the diagnosis of BrS. While the type 1 pattern is usually associated with cases of SCA, it can often masquerade as BrP type 2 upon workup [11]. Interestingly, the eventual diagnosis of a BrP type 1 is usually clinically divulged in the aftermath of concomitant infection, such as pneumonia. Similarly, in our case, our patient initially presented with a typical type $2 \mathrm{BrP}$ but was later also found to be positive for type 1 in the wake of the ajmaline (sodium blocker) challenge test.

\section{Treatment}


Treatment for BrS is centered around the prevention of ventricular arrhythmias and SCA through the means of an ICD. An ICD is indicated in patients with documented electrophysiological ailments, survivors of SCA, and patients with a family history of SCA. Patients with BrP do not require any specific therapy in the absence of strong family history and an electrophysiology study provoking ventricular arrhythmias. ICD is extremely safe and highly efficacious in terminating ventricular arrhythmias in patients with SCA. It is, therefore, an essential component of the treatment regimen [12].

\section{Conclusions}

BrS can initially present as type $2 \mathrm{BrP}$ with SCA. Initial ECG findings can be unremarkable, and frequent ECGs should be performed during exposure to exacerbating risk factors such as pneumonia, as these circumstances can unmask the pathognomonic findings of a type $2 \mathrm{BrP}$. Confirmatory test with ajmaline challenge should be done for BrP type 2 to find any type 1 pattern, as this would increase the risk of future SCA. Treatment with ICD placement is preferred to prevent future SCA in patients with diagnosed BrS.

\section{Additional Information \\ Disclosures}

Human subjects: Consent was obtained by all participants in this study. Conflicts of interest: In compliance with the ICMJE uniform disclosure form, all authors declare the following: Payment/services info: All authors have declared that no financial support was received from any organization for the submitted work. Financial relationships: All authors have declared that they have no financial relationships at present or within the previous three years with any organizations that might have an interest in the submitted work. Other relationships: All authors have declared that there are no other relationships or activities that could appear to have influenced the submitted work.

\section{References}

1. Brugada P, Brugada J: Right bundle branch block, persistent ST segment elevation and sudden cardiac death: a distinct clinical and electrocardiographic syndrome: a multicenter report. J Am Coll Cardiol. 1992, 20:1391-1396. 10.1016/0735-1097(92)90253-j

2. Sorajja D, Ramakrishna H, Poterack AK, Shen WK, Mookadam F: Brugada syndrome and its relevance in the perioperative period. Ann Card Anaesth. 2015, 18:403-413. 10.4103/0971-9784.159812

3. Bernardo $\mathrm{MH}$, Tiyyagura SR: A case of type I and II Brugada phenocopy unmasked in a patient with normal baseline electrocardiogram (ECG). Am J Case Rep. 2018, 19:21-24. 10.12659/AJCR.906464

4. Veltmann C, Schimpf R, Echternach C, et al.: A prospective study on spontaneous fluctuations between diagnostic and non-diagnostic ECGs in Brugada syndrome: implications for correct phenotyping and risk stratification. Eur Heart J. 2006, 27:2544-2552. 10.1093/eurheartj/ehl205

5. Keller DI, Rougier JS, Kucera JP, et al.: Brugada syndrome and fever: genetic and molecular characterization of patients carrying SCN5A mutations. Cardiovasc Res. 2005, 67:510-519. 10.1016/j.cardiores.2005.03.024

6. Nagase S, Kusano KF, Morita H, et al.: Longer repolarization in the epicardium at the right ventricular outflow tract causes type 1 electrocardiogram in patients with Brugada syndrome. J Am Coll Cardiol. 2008, 51:1154-1161. 10.1016/j.jacc.2007.10.059

7. Yan GX, Antzelevitch C: Cellular basis for the Brugada syndrome and other mechanisms of arrhythmogenesis associated with ST-segment elevation. Circulation. 1999, 100:1660-1666. 10.1161/01.cir.100.15.1660

8. Priori SG, Wilde AA, Horie M, et al.: HRS/EHRA/APHRS expert consensus statement on the diagnosis and management of patients with inherited primary arrhythmia syndromes: document endorsed by HRS, EHRA and APHRS in May 2013 and by ACCF, AHA, PACES, and AEPC in June 2013. Heart Rhythm. 2013, 10:19321963. 10.1016/j.hrthm.2013.05.014

9. Bayes de Luna A, Brugada J, Baranchuk A, et al.: Current electrocardiographic criteria for diagnosis of Brugada pattern: a consensus report. J Electrocardiol. 2012, 45:433-442. 10.1016/j.jelectrocard.2012.06.004

10. Wilde AA, Antzelevitch C, Borggrefe M, et al.: Proposed diagnostic criteria for the Brugada syndrome: consensus report. Circulation. 2002, 106:2514-2519. 10.1161/01.cir.0000034169.45752.4a

11. Makarawate P, Chaosuwannakit N, Vannaprasaht S, Tassaneeyakul W, Sawanyawisuth K: Clinical characteristics and treatment outcomes of patients with Brugada syndrome in northeastern Thailand. Singapore Med J. 2014, 55:217-220. 10.11622/smedj.2014055

12. Antzelevitch C, Fish J: Therapy for the Brugada syndrome. Basis and Treatment of Cardiac Arrhythmias. Handbook of Experimental Pharmacology. Springer, Berlin, Heidelberg; 2006. 171:305-330. 